\title{
Estimación del riesgo cardiovascular en pacientes con angina crónica estable
}

Clayton, et. al. BMJ 2005; 331:869-72.

\section{Objetivo}

Predecir el riesgo de accidente cerebrovascular (ACV) incapacitante, infarto agudo de miocardio (IAM) o muerte por cualquier causa en pacientes con angina crónica estable (ACE) y función sistólica preservada.

\section{Diseño}

Anális a partir de una base de datos de un ensayo clínico aleatorizado.

Lugar

Inglaterra, Hungría, Holanda, Israel, Canadá y Nueva Zelanda.

\section{Pacientes}

7311 pacientes con ACE bajo tratamiento médico y que tuvieran IAM previo o coronariopatía demostrada por cateterismo.

\section{Medición de resultados principales}

Muerte por cualquier causa, IAM o ACV incapacitante durante un seguimiento promedio de 4,9 años.

\section{Resultados}

Se obtuvo información sobre 16 variables fácilmente recabables en la práctica clínica (ver en el cuadro 1 las de significancia estadística). El punto final combinado ocurrió en 1063 pacientes. El riesgo a cinco años de sufrir un evento osciló entre $4 \%$ en el decilo inferior del puntaje hasta $35 \%$ en el superior.
Cuadro 1: riesgo asociado a IAM, ACV incapacitante y muerte de los predictores con significancia estadística.

\begin{tabular}{|c|c|}
\hline & Muerte, AM o ACV \\
\hline Edad (por cada diez años a partir de los 60 ) & $1,73(1,57$ a 1,92$)$ \\
\hline Fracción de eyección (por cada $5 \%$ de cada a partir de los 60 años de edad) & $1,19(1,13$ a 1,25$)$ \\
\hline Tabaquismo actual & $1,82(1,57$ a 2,2$)$ \\
\hline Recuento de glóbulos blancos & $1,07(1,05$ a 1,09$)$ \\
\hline Diabetes & $2,33(1,74$ a 3,14$)$ \\
\hline Hlperglucemia (por cada 100mg/dl de elevación a partir de 100mg/dL) & $1,08(1,04$ a 1,11$)$ \\
\hline Creatininemia (por cada 0,1mg/dll de elevación a partir de 1,5mg/dL) & $1,08(1,04$ a 1,12$)$ \\
\hline Antecedente de ACV & $1,7(1,27$ a 2,28$)$ \\
\hline Al menos un ataque anginoso por semana & $1,25(1,1$ a 1,42$)$ \\
\hline Compromiso de tres o mas vasos (si se realizó cinecoronariografía) & $1,28(1,1$ a 1,5$)$ \\
\hline Sin tratamiento hipolipemiante & $1,23(1,08$ a 1,4$)$ \\
\hline Intevalo QT mayor a $450 \mathrm{~ms}$ & $1,26(1,08$ a 1,45$)$ \\
\hline Presión arterial sistólica mayor a 150 mmHg. & $1,23(1,07$ a 1,42$)$ \\
\hline Por cada droga antianginosa adicional & $1,14(1,04$ a 1,25$)$ \\
\hline Infarto previo & $1,15(1,01$ a 1,3$)$ \\
\hline
\end{tabular}

\section{Conclusión}

Esta estimación de riesgo resulta una herramienta útil en la práctica clínica ambulatoria para la toma de decisiones en el manejo de pacientes con ACE, a fin de disminuir la incidencia de eventos adversos graves en el mediano plazo.

Fuente de financiamiento: Bayer Healthcare AG, Wupertal, Alemania

\section{Comentario}

Los datos de este modelo de predicción provienen del primer ensayo clínico de grandes dimensiones para eventos duros, todos inequívocos ya que no se incluyeron infartos definidos sólo por la elevación de troponina, lo que le confiere a esta regla mayor relevancia y aplicación clínica. No hubo relación entre la tasa de utilización de procedimientos de revascularización como tratamiento de la angina y el riesgo de padecer eventos duros, lo que sugiere que los eventos graves están mayormente vinculados a accidentes de placa, con fisiopatología y factores de riesgo diferentes de aquellos que determinan la progresión subaguda o crónica de la angina. La tasa de eventos a cinco años fue tan sólo 4,7\%, llamativamente baja si consideramos que en los estudios HOPE $^{1}$, EUROPA ${ }^{2}$ y $\mathrm{PEACE}^{3}$, todos con pacientes de un perfil similar, fue 15,10 y $8 \%$ respectivamente. Esta comparación sugiere que se trata de una población extremadamente seleccionada, lo que lleva a preguntarse cuál será la aplicabilidad de este puntaje en los pacientes de alto riesgo del mundo real. Este modelo no fue probado en una cohorte de pacientes distinta de aquella empleada para diseñarlo, lo que le resta validez externa. Respecto de su implementación, a estar compuesto por 16 variables, su aplicación depende de un cálculo engorroso que no hemos reproducido. Afortunadamente, está disponible un sitio web (www.anginarisk.org) que agiliza este cálculo y lo vuelve más accesible. Finalmente, los autores concluyen que, dado que la revascularización prolonga la sobrevida sólo en pacientes de alto riesgo, este puntaje ayudará a identificar a aque- llos pacientes con angina estable que más se beneficiarán con este procedimiento. Esta conclusión es discutible. Por un lado, el alto riesgo en que se apoya esa afirmación está dado por variables como la presencia de insuficiencia cardíaca (IC), un área isquémica extensa, una fracción de eyección deteriorada o una mala clase funcional $(\mathrm{CF})^{4}$, variables que no fueron incluidas en el modelo en cuestión. Y por otro lado, un paciente añoso con creatininemia elevada, fumador, hipertenso, con antecedente de IAM y ACV tiene alto riesgo de morir, pero probablemente no se beneficie con una revascularización. El punto crucial es si discriminar el riesgo en este grupo de pacientes cambia el enfoque terapéutico. Es nuestra impresión que, a la luz de la evidencia actual, el uso de aspirina ${ }^{5}$, estatinas a altas dosis ${ }^{6}$ e IECA se recomienda a casi la totalidad de los pacientes coronarios crónicos; mientras que la revascularización se indicará -más allá de los resultados de este puntajesegún la clase funcional, la progresión de síntomas, la presencia de IC, la magnitud del área isquémica y la función ventricular.

\section{Conclusión del comentador}

si bien este puntaje discrimina bien el riesgo en pacientes con angina crónica estable, no queda claro que cambie el enfoque terapéutico ya que la mayoría debe recibir el mismo tratamiento médico, conservando la revascularización indicaciones precisas e independientes.

* ver glosario

Mariano Trevisan [ Médico Especialista en Cardiología. Servicio de Cardiología. Sanatorio San Carlos. San Carlos de Bariloche, Río Negro. ]

Trevisan M. Un puntaje ayuda a dicriminar el riesgo cardiovascular en pacientes con angina crónica estable. Evid. actual. páct. ambul. 9(2);42. Mar-Abr 2006. Comentado de: Clayton TC, Lubsen J, Pocock SJ. Risk score for predicting death, myocardial infarction, and stroke in patients with stable angina, based on a large randomised trial cohort of patients. BMJ 2005; 331:869-72. PMID 16210253

\section{Referencias}

1. The Heart Outcomes Prevention Evaluation Study Investigators. Effects of an angiotensin-converting-enzyme inhibitor, ramipril, on cardiovascular events in high risk patients. N Engl J Med 2000;342:145- 53

2. Fox KM. Efficacy of perindopril in reduction of cardiovascular events among patients with stable coronary artery disease: randomised, double-blind, placebocontrolled, multicentre trial (the EUROPA study). Lancet 2003;362:782-8.

3. The PEACE Trial Investigators. Angiotensin-converting- enzyme inhibition in stable coronary artery disease. N Engl J Med 2004;351:2058-68.

4. O'Toole L, Grech ED. Chronic stable angina: treatment options. BMJ. 2003 May 31;326(7400):1185-8.

5. Antiplatelet Trialists' Collaboration. Collaborative overview of randomised trials of antiplatelet therapy--I: Prevention of death, myocardial infarction, and stroke by prolonged antiplatelet therapy in various categories of patients. BMJ. 1994 Jan 8;308(6921):81-106

6. LaRosa JC, Grundy SM, Waters DD, et al. Intensive lipid lowering with atorvastatin in patients with stable coronary disease.N Engl J Med 2005;352:1425-35. 